\title{
Cirugía oncoplástica de la mama: Cómo mejorar la resecabilidad en cirugía conservadora asegurando la estética*
}

\author{
Drs. RODRIGO IGLESIS G. ${ }^{1}$, RODRIGO CABELLO P. ${ }^{1}$, \\ MONTSERRAT FONTBONA T. ${ }^{1}$, RAMÓN BAEZA S. ${ }^{2}$, BRUNO DAGNINO ${ }^{1}$ \\ Servicio de Cirugía Oncológica y Plástica. Hospital Militar de Santiago. \\ 2 Instituto de Radiomedicina (IRAM).
}

\begin{abstract}
\section{Oncoplastic surgery for breast cancer}

Background: In breast cancer surgery, the association of reductive mammoplasty to oncological excision, allows more extensive excisions, reducing radical surgery and preventing secondary deformities. This is called oncoplastic or tumor specific mammary reconstruction. Aim: To report 37 patients in whom oncoplastic surgery was used. Material and Methods: Retrospective descriptive series of 37 patients aged 38 to 65 years, subjected to oncoplastic mammary surgery, that were followed for a median of 42 months. Results: Eighty six percent considered the cosmetic result of surgery as satisfactory. One patient had a local relapse associated to systemic dissemination at 16 months. One patient died and one is under hormonal treatment for bone metastases. No significant complications or delays in oncological therapies were recorded. Conclusions: Oncoplastic surgery may avoid mastectomy and prevent deformities in breast cancer surgery.
\end{abstract}

Key words: Oncoplastic surgery, breast cancer, mastectomy.

\section{Resumen}

Conservar la mama se considera uno de los objetivos importantes en el tratamiento del cáncer. Frecuentemente la relación entre el tamaño y la localización tumoral impide la cirugía conservadora con un buen resultado estético u obliga a realizar una mastectomía, lo que empobrece la calidad de la sobrevida. Asociar técnicas de mamoplastía reductiva a la resección oncológica ha permitido aumentar la extensión de las resecciones, disminuyendo las cirugías radicales o previniendo las deformidades secundarias. Es lo que llamamos oncoplástica mamaria (OPM) o reconstrucción mamaria tumor específica. Presentamos una serie descriptiva retrospectiva de 37 pacientes en quienes usamos OPM como alternativa a la mastectomía. Un $86 \%$ consideró el resultado cosmético como satisfactorio. La mediana de seguimiento fue 42 meses. Una paciente ha tenido recurrencia local asociada a diseminación sistémica a los 16 meses. Una paciente falleció y otra está en tratamiento hormonal por metástasis óseas. No hubo complicaciones importantes en la serie ni retardo significativo de las terapias secuenciales. Concluimos que esta técnica permite evitar mastectomías y prevenir deformidades en cirugía conservadora estándar.

Palabras clave: Mastectomía, mamoplastía reductiva, cáncer de mama.

*Recibido el 31 de Marzo de 2009 y aceptado para publicación el 25 de Julio de 2009.

Correspondencia: Dr. Rodrigo Iglesis G.

Avda. Larraín 9100, Santiago, Chile

E-mail: riglesis@hotmail.com 


\section{Introducción}

La conservación de la glándula se considera uno de los objetivos importantes en el tratamiento del cáncer de mama ${ }^{1}$. El concepto de tratamiento conservador implica remoción completa del tumor con un margen concéntrico de tejido sano y realizado de una forma cosméticamente aceptable ${ }^{2}$. Sin embargo, frecuentemente la relación entre el tamaño y la localización tumoral (que determina la cantidad de tejido a resecar en la obtención de un límite libre de lesión) y el volumen mamario residual impide la cirugía conservadora con un buen resultado estético, u obliga a realizar una mastectomía. Ambas situaciones empobrecen la calidad de la sobrevida ${ }^{3,4}$. El asociar técnicas de mamoplastía reductiva a la resección oncológica y, eventualmente simetrizando la mama contralateral, ha permitido aumentar la extensión de las resecciones en casos seleccionados disminuyendo las cirugías radicales o previniendo deformidades secundarias. Es lo que llamamos oncoplástica mamaria $(\mathrm{OPM})$ o reconstrucción mamaria tumor específica ${ }^{5-7}$.

Presentamos una serie descriptiva retrospectiva de pacientes en quienes usamos OPM como alternativa a la mastectomía.

\section{Material y Método}

Treinta y siete mujeres con edad entre 38 y 65 años consintieron informadamente ser tratadas quirúrgicamente por cáncer mamario entre julio de 1998 y septiembre de 2007 con terapia conservadora usando alguna técnica oncoplástica, suponiendo que el estándar quirúrgico produciría deformidad importante secundaria, o no era posible.

Un tumor filodes maligno recidivado, seis carcinomas in situ extensos y 30 infiltrantes: 22 T2 y $8 \mathrm{~T} 3$, tres de los cuales habían recibido neoadyuvancia con Adriamicina (Tabla 1).

Se usaron diferentes técnicas de mamoplastías reductivas, dependiendo de la resección oncológica: En 13 periareolar y en las restantes 24, 12 pe-

Tabla 1. Tipo histológico

\begin{tabular}{lr} 
& n $\mathbf{3 7}$ \\
\hline Tumor filodes maligno & 1 \\
Carcinoma ductal in situ extenso & 5 \\
Carcinoma ductal in situ con microinvasión & 26 \\
Carcinoma ductal infiltrante & 4 \\
Carcinoma lobulillar infiltrante & 1 \\
\hline
\end{tabular}

dículo superior o inferior tipo McKissock, 6 superomedial y 6 con tipo I de Ribeiro, pexia con resección de piel según patrón de Weis ${ }^{7}$. En tres debió resecarse el complejo areola pezón (CAP). El examen histopatológico intraoperatorio aseguró resecciones con límites libres de neoplasia y el lecho operatorio se marcó con clips. La axila se manejó en forma habitual. En 24 pacientes se simetrizó la mama contralateral sincrónicamente. Todas ellas recibieron radioterapia externa a mama, y cuando estaba indicado, a linfáticos periféricos, con dosis y fraccionamiento estándar: $5.000 \mathrm{cGy}$ en 5 semanas iniciada entre 4 y 7 semanas después de la cirugía.

El seguimiento mínimo fue de 6 meses, con una mediana de 42 meses.

Los resultados estéticos fueron evaluados con criterios sugeridos por Kroll y Strasser ${ }^{8,9}$, considerando simetría, proyección y forma. En cuanto a morbilidad se consideró la necrosis de piel con retardo de cicatrización y del CAP, infección mayor, colecciones que requirieron drenaje, necesidad de reoperación e hiperpigmentación.

\section{Resultados}

En todas las pacientes en que se planificó el procedimiento oncoplástico éste pudo realizarse. $\mathrm{La}$ mediana del peso de los especímenes quirúrgicos fue de 124 grs. A la fecha un paciente de la serie presentó recurrencia local (1 de 37, 2,7\%) asociada a diseminación pulmonar y ósea de la enfermedad 16 meses post cirugía. Una paciente falleció por diseminación sistémica a los seis años y una se encuentra con metástasis óseas en tratamiento hormonal. No hubo pérdida importante de piel o del CAP en la mama tratada o cuando se simetrizó la mama contralateral. Seis pacientes tuvieron retardo de cicatrización en la unión de la cicatriz en $\mathrm{T}$ in-

Tabla 2. Complicaciones

\begin{tabular}{lcc}
\hline & $\begin{array}{c}\text { Mama } \\
\text { con Ca. }\end{array}$ & $\begin{array}{c}\text { Mama } \\
\text { contralat. }\end{array}$ \\
\hline Necrosis de piel & 1 & 0 \\
Retardo cicatrización & 6 & 4 \\
Necrosis parcial CAP & 2 & 1 \\
Necrosis total CAP & 0 & 0 \\
Hematoma & 2 & 1 \\
Celulitis & 1 & 1 \\
Seroma infectado & 2 & 0 \\
Hiperpigmentación importante & 4 & 0 \\
\hline
\end{tabular}


vertida, lo que retardó en dos a tres semanas el inicio de su terapia oncológica secuencial (Tabla 2). Un $86 \%$ considera el resultado cosmético como bueno o excelente y se considera satisfecha con él (Tabla 3) (Figuras 1, 2 y 3). No se encontró neoplasia en las muestras de mama contralateral.
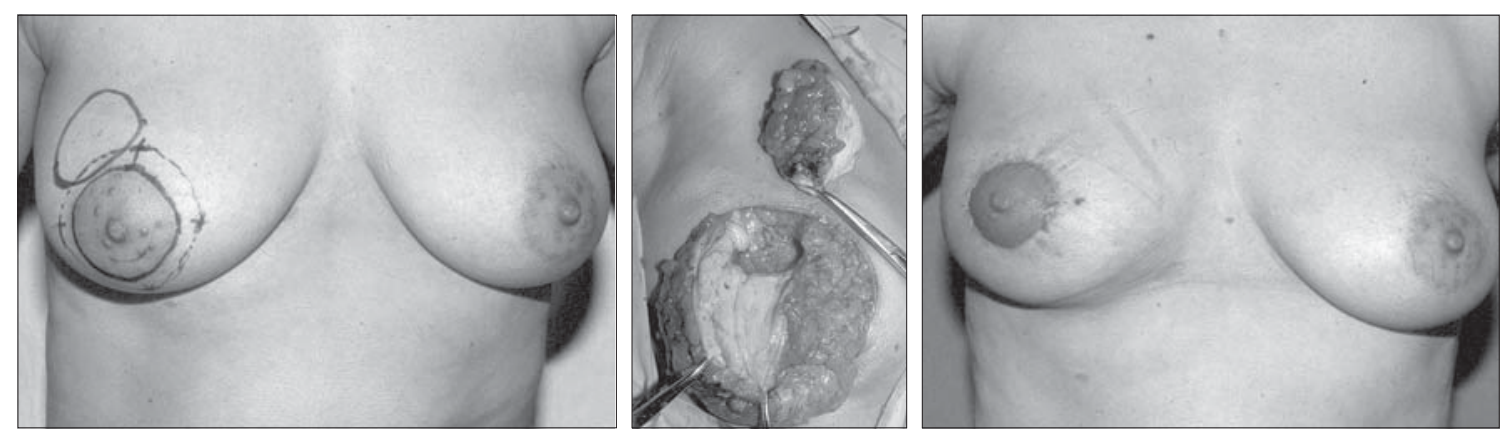

Figura 1. Treinta y ocho años, tumor $4,5 \mathrm{~cm}$ cuadrante superior externo derecho. Con técnica periareolar se ha decolado toda la mama y practicado tumorectomia y disección axilar por la misma incisión. Resultado 8 meses post radioterapia.
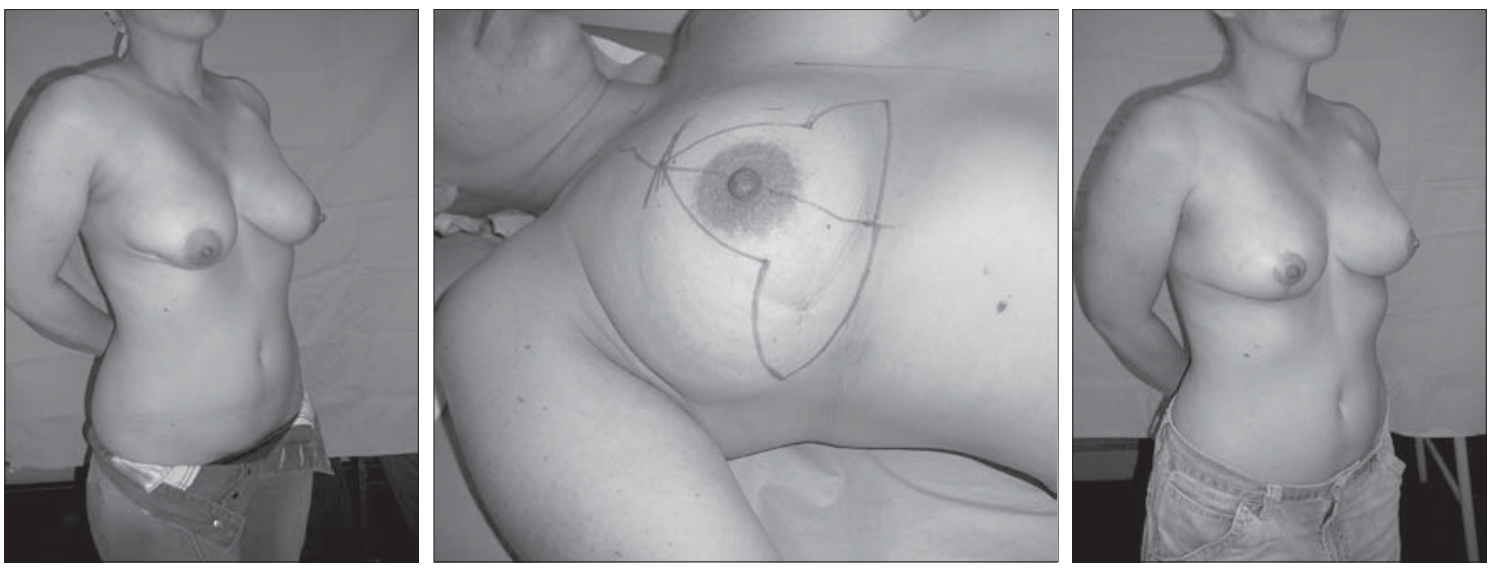

Figura 2. Cuarenta años, con tumor $6 \mathrm{~cm}$ cuadrante inferior externo y compromiso de piel. Neoadyuvancia con 4 ciclos AC (izq). Marcación de la piel a resecar (centro). Resultado con simetrización mama contralateral (der).
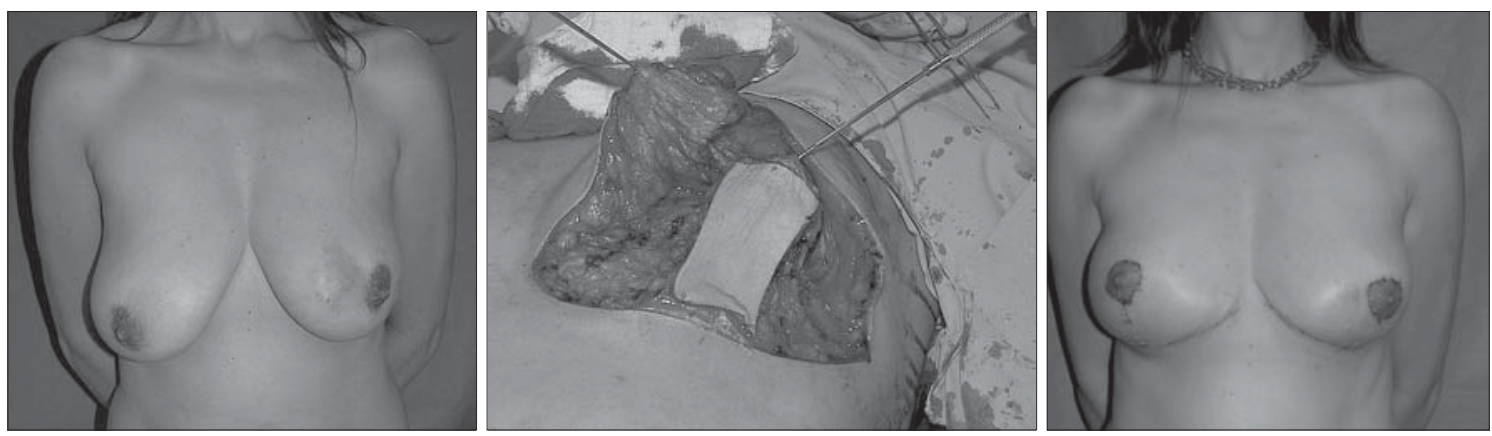

Figura 3. Treinta y seis años, con extenso cáncer mama (izq). Reseccion de todo el cuadrante inferior interno y parte del superior con reconstrucción con pedículo inferior (centro). Simetrización de la mama contralateral (der). 


\section{Discusión}

Cada día se ha hecho más trascendente la conservación de la glándula en el tratamiento del cáncer de mama. La cirugía conservadora, seguida de radioterapia, ha reemplazado a los tratamientos radicales con sobrevida a 20 años sin diferencias estadísticamente significativas ${ }^{10,11}$. Sin embargo, en más de un tercio de los casos la técnica "estándar" no logra márgenes de resección adecuados ${ }^{12}$, lo que aumenta significativamente las recidivas locales y/o se obtienen malos resultados cosméticos, que se traducen en insatisfacción de las pacientes ${ }^{8,2}$. Múltiples causas explican esta falla estética: resecciones extensas que producen distorsión, retracción y notorios cambios en el volumen de la mama operada, como también cambios en la posición del complejo areola-pezón que se traducen en asimetrías que suelen ser muy perturbadoras para la mujer. La integración de las técnicas de mamoplastía reductiva al tratamiento conservador del cáncer de mama (oncoplástica mamaria) descritas por Audresch y Petit han permitido mejorar estas situaciones ${ }^{5,6}$.

La técnica oncoplástica a usar depende de la extensión y principalmente de la localización del tumor. Lesiones de cuadrantes inferiores o centrales en que los defectos con tumorectomía son más frecuentes y evidentes, se benefician sustancialmente con técnicas reductivas de pedículo superomediales, con resecciones de piel más extensas y cicatriz en ancla. Las lesiones de polo superior pueden abordarse más fácilmente con técnica periareolar, o bien con técnicas de pedículo inferior que aseguren adecuada irrigación del complejo areola pezón ${ }^{13,14}$

La simetrización sincrónica de la mama contralateral, cuando es necesaria, permite que la paciente obtenga buen resultado estético sin necesidad de procedimientos correctivos posteriores, lo que significa un ganancial en su estado anímico, y por otra parte, permite examinar el tejido de la mama contralateral sabiendo que la incidencia de cáncer es mayor en estas pacientes. Rietjens reporta hasta $4,3 \%$ de cáncer contralateral en mujeres tratadas con OPM y simetrización sincrónica ${ }^{14}$.

El seguimiento clínico y por imágenes no nos ha significado dificultades adicionales con esta técnica. Si bien nuestra serie es pequeña y tiene seguimiento medio de sólo 40 meses, la falla local y a distancia se asemeja a las series internacionales publicadas $^{15-18}$ que reportan recurrencia local a 5 años menores al $8 \%$ y sobrevida global de $86 \%$. Junto con esto se mantiene un alto grado de satisfacción de las pacientes con el procedimiento. Es interesante destacar que estas cifras de satisfacción se obtuvieron en una población de pacientes que recibió radioterapia post operatoria. Estos resultados coinciden con nuestra experiencia publicada en situaciones similares ${ }^{19,20}$.

El peso promedio de 124 gr de los especímenes operatorios ratifica lo extenso de las resecciones. A pesar de ser tumores grandes, por lo general se obtiene márgenes más seguros con estas técnicas que disminuyen la probabilidad de recurrencia lo$\mathrm{cal}^{21}$. Kaur et al, reportaron un estudio retrospectivo no randomizado que, al comparar el estado de los márgenes quirúrgicos y volúmenes de resección en cirugía oncoplástica con la cuadrantectomía estándar, en las primeras eran mayores y con márgenes más alejados ${ }^{22}$.

\section{Conclusión}

El uso de técnicas reductivas de cirugía plástica mamaria asociadas a la resección oncológica, permite en casos seleccionados, aumentar las indicaciones de la cirugía conservadora en cáncer de mama previniendo deformidades secundarias o la mastectomía, manteniendo la seguridad oncológica.

\section{Referencias}

1. II Jornada Chilena de Consenso en Cáncer de Mama. Viña del Mar. Septiembre 2003.

2. Schwartz GF, Veronesi U, Clough KB, Dixon JM, Fentiman IS, Heywang-Köbrunner SH, et al: Proceedings of the consensus conference on breast conservation, Milan, Italy. Int J Radiation Oncology Biol Phys 2006; 65: 1281-1288.

3. Ferrell B, Wisdom, CH, Wenzl C. Quality of live as an outcome variable in the management of cancer patient. Cancer 1989, 63: 2321-2327.

4. Al-Ghazal SK, Fallowfield L, Blamey RW. Comparison of psychological aspect and patient satisfaction following breast conservation surgery, simple mastectomy and breast reconstruction. Eur J Cancer 2000; 36: 1938-1943.

5. Audretsch W, Rezai M, Kolotas C, Zamboglou N, Schnabel T. Tumor-specific inmediate reconstruction in breast cancer patient. Perspectives in Plastic Surgery 1998; 11: 71-106.

6. Petit JY, Rietjens M, Garusi C, Greuze M, Perry C. Integration of plastic surgery in the course of breast conservation surgery for the cancer treatment to improve cosmetic result and radicality of tumor excision. Recent Result of Cancer Research 1998; 152: 202-211.

7. Bostwick III, John. Plastic And Reconstructive Breast Surgery. 2nd Edition. Hardcover, 1999.

8. Strasser EJ. Application of an Objective Grading System for the Evaluation of Cosmetic Surgical Results. Plast 
Reconstr Surgery 2002; 109: 1733-1740.

9. Kroll SS, Baldwin B. A Comparison of Outcomes Using three different methods of breast reconstruction. Plastic Reconstr Surg 1992; 90: 455-462.

10. Verones U, Cascinelli N, Mariani L, Greco M, Saccozzi $\mathrm{R}$, Luini A, et al. Twenty-year follow-up of a randomized study comparing breast-conserving surgery with radical mastectomy for early breast cancer. $\mathrm{N}$ Engl J Med 2002; 347: 1227-1232.

11. Gordon F, Schwartz U, Veronesi KB, Clough J, Dixon $\mathrm{C}$, Fentiman SH, et al: Proceedings of the consensus conference on breast conservation. Int $\mathrm{J}$ Radiation Oncology Biol Phys 2006; 65: 1281-1288.

12. Audretsch WP. Reconstruction of the partial mastectomy defect: classification and method. In: Spear SL, Willey SC, Robb GL, et al, editors. Surgery of the breast: principles and art. Philadelphia: Lippincott Williams \& Wilkins; 2006; pp 179-216.

13. Silverstein MJ, Gierson ED, Colburn WJ, Cope LM, Furmanski M, Senofsky GM, et al. Can intraductal carcinoma be excised completely by local excision? Clinical and pathological predictors. Cancer 1994; 73 : 2985-2989.

14. Rietjens M, Petit JY, Contesso J, Bertin F, Gilles R. The role of reduction mammaplasty in oncology. Eur J Plastic Surg 1997; 20: 246-250.

15. Clough KB, Cuminet J, Fitoussi A, Nos C, Mosseri V. Cosmetic sequelae after conservative treatment for breast cancer: classification and result of surgical correction. Ann Plast Surg 1998; 41: 471-481.

16. Krishna B, Clough JS, Lewis B, Couturaud A, Fitoussi C, Nos MC. Oncoplastic Techniques Allow Extensive Resections for Breast-Conserving Therapy of Breast Carcinomas. Ann Surg 2003; 237: 26-34.

17. Cothier-Savey I, Otmezguine Y, Calitchi E, Sabourin JC, Le Bourgeois JP, Baruch J. Value of reduction mammoplasty in the conservative treatment of breast neoplasms. A propos of 70 cases. Ann Chir Plast Esthet 1996; 41: 346-353.

19. Iglesis R, Cabello R, Lee K, Baeza R: ¿Es la radioterapia contraindicación para la reconstrucción mamaria inmediata con tejido antólogo? Rev Chil Cancerología y Hematología 2006; 15: 161-164.

20. Iglesis, R, Cabello R, Fontbona M, Lee K, Baeza R: Mastectomía parcial amplia y reconstrucción inmediata con tejido autólogo. Indicaciones, técnica y resultados. Rev Chil Cancerología y Hematología 2007; 16: 153-158.

21. Vicini FA, Kestin LL, Goldstein NS. Relationship between excision volume, margin status, and tumor size with the development of local recurrence in patients with ductal carcinoma in-situ treated with breast-conserving therapy. J Surg Oncol 2001; 76: 245254.

22. Kaur N, Petit JY, Rietjens M, Maffini F, Luini A, Gatti G, et al. Comparative study of surgical margins in oncoplastic surgery and quadrantectomy in breast cancer. Ann Surg Oncol 2005; 12: 539-545. 